\title{
Clinical and Radiographic Variables Around Implant With Simultaneous Graft Among Type 2 Diabetic Patients Treated With Different Hypoglycemic Medications: A Retrospective Study.
}

\section{Shaojie Shi}

Air Force Medical University

\section{Feng Ding}

Air Force Medical University

Xiangdong Liu

Air Force Medical University

Lei Wang

Air Force Medical University

\section{Xingxing Wang}

Air Force Medical University

Sijia Zhang

Air Force Medical University

Guoqiang Zhao

Air Force Medical University

Yingliang Song ( $\nabla$ songyingliang@163.com )

Air Force Medical University

\section{Research Article}

Keywords: type 2 diabetic mellitus, dental implant, marginal bone loss, hypoglycemic agents, guided bone regeneration

Posted Date: December 31st, 2020

DOl: https://doi.org/10.21203/rs.3.rs-134530/v1

License: (c) (i) This work is licensed under a Creative Commons Attribution 4.0 International License.

Read Full License 


\section{Abstract}

Background: The clinical and radiographic variables around dental implants in type 2 diabetes mellitus patients with different hypoglycemic agents still remained unclear.

Methods: This retrospective cohort study collected the dental records and digital periapical radiographs of type 2 diabetes mellitus patients and implants. These patients were grouped according to their medication: insulin, metformin, and glucagon-like peptide-1 drugs. The radiographic marginal bone loss around implants and clinical parameters, including peri-implant bleeding on probing and probing depth, were compared among groups using the Kruskal-Wallis test.

Results: A total of 150 patients with 308 implants (101 in insulin group, 121 in metformin group and 86 in glucagon-like peptide-1 drugs group) were assessed. The peri-implants marginal bone loss in insulin group $(P<0.05)$ and metformin group $(P<0.01)$ were significantly higher than glucagon-like peptide-1 drug group. The radiographic bone loss in metformin was higher than insulin group $(P<0.05)$. While there was no statistical difference of clinical peri-implant parameters among groups $(P>0.05)$.

Conclusions: The radiographic variables were not exactly the same among type 2 diabetes mellitus patients with different hypoglycemic agents. glucagon-like peptide- 1 drugs might be more beneficial to bone tissue around implants. More studies are needed to verify the direct effect of these drugs on periimplant bone.

Clinical trial registration number: ChiCTR2000034211 (retrospectively registered)

\section{Background}

The number of individuals with type 2 diabetes mellitus (T2DM) constitutes a large population, and a recent report in 2019 showed that, the prevalence of T2DM worldwide had reached $8 \%$ in $2018^{1}$. Due to the relationship between T2DM and periodontitis ${ }^{2}$, there is a large proportion of patients with missing teeth in the T2DM population. Implant-supported denture restoration has been an excellent treatment for the loss of teeth and T2DM individuals have a large requirement for dental implant treatment. However, T2DM patients often face the challenge of atrophic alveolar bone width at implant sites ${ }^{3}$. The minor and moderate atrophic ridge often need horizontal bone augmentation by guided bone regeneration (GBR)with the combination of bone graft materials and barrier membranes ${ }^{4}$. The uncontrolled hyperglycemia would impact the therapeutic effect of dental implants in T2DM ${ }^{5}$. And some studies have shown that early osseointegration around implants is compromised by T2DM even if glucose is strictly controlled $^{6}$. It is crucial for clinicians to promote the efficacy of implant treatment in T2DM patients.

Dental implant treatment success is related to the osseointegration and peri-implant bone around implants $^{7-8}$. Previous research has shown that diabetes impacts bone remodeling around implants, especially at the early stage of osseointegration ${ }^{9}$. The changes of bone levels around implants is a 
biomechanical process that could be influenced by several factors in patients and implants ${ }^{10}$. The marginal bone loss (MBL) in diabetes patients is higher than that in nondiabetic individuals, despite being glycemic controlled ${ }^{11}$. The higher MBL in T2DM patients could reflect the impaired bone condition around implants. Therefore, MBL was considered an important radiographic parameter for bone tissue around implants in the present study.

Recently, abundant evidence has revealed the role of hypoglycemic agents in bone metabolism ${ }^{12}$. There is growing concern about their direct bone target effect. For example, insulin and metformin could be beneficial to bone tissue ${ }^{13}$. This beneficial effect might improve the outcome of dental implants. It was reported that local application of insulin could promote osseointegration in T2DM rats ${ }^{14}$. Glucagon-like peptide-1 (GLP-1) drugs also have the potential to promote osseointegration around implants ${ }^{15}$. Currently, there is no clinical evidence of hypoglycemic drugs interfering with bone tissue around implants. Therefore, the present study aimed to obtain more detailed information about the clinical and radiographic variables around implants in T2DM patients using different hypoglycemic agents.

\section{Methods}

\section{Ethical protocol}

The retrospective cohort study was approved by the Ethics Committee of School of Stomatology, the Fourth Military Medical University (Ethics Approval Number: IRB-REV-2020045) and in compliance with the Helsinki Declaration. All patients were given complete information about the treatment and signed informed consent forms before surgery. The implant treatment was performed by the same doctor and the trauma was minimized. This study is reported in agreement with the STROBE (Strengthening the Reporting of Observational Studies in Epidemiology) statement.

\section{Study designand setting}

This retrospective cohort study was designed to compare the clinical and radiographic variables around implants with simultaneous GBR in T2DM patients using different hypoglycemic agents. The examiners collected dental records of T2DM patients who received implant surgery from January 2015 to November 2019. After information collection, one examiner divided them into three groups based on their major hypoglycemic agent species: insulin (group 1), metformin (group 2), and GLP-1 (group 3). The state immediately after surgery was recorded as baseline. The MBL from immediately after implant placement to before prosthetic installation and to the first-year follow-up after functional loading were measured to suggest the change of bone tissue around implants. The clinical inflammatory parameters at the firstyear follow-up after functional loading, including bleeding on probing (BOP) and probing depth (PD), were collected. To ensure the principle of blinding, the groups were sealed and another independent examiner assessed the data among groups.

\section{Eligibility criteria}


The inclusion criteria were as follows: (a) medically diagnosed T2DM; (b) good blood glucose control before surgery $\left(\mathrm{HbA} 1 \mathrm{c} \% \leq 8 \%{ }^{16}\right)$; (c) treatment with one of the hypoglycemic agents: metformin, insulin or GLP-1; (d) implant site with Seibert class I ridge deficiencies resulting in the implant thread exposure which treated only with GBR (graft mass $\leq 0.25 \mathrm{~g}$ ); (e)good oral hygiene care with regular semidiurnal brushing and semiannual professional cleaning.

The exclusion criteria were as follows: (a) poor glycemic control ( $\mathrm{HbA} 1 \mathrm{c} \%>8 \%)$ or severe diabetic complications; (b) osteoporosis; (c) long-term use of bisphosphonates or steroids; (d) irregular use of hypoglycemic drugs; (e) excessive tobacco use and alcohol consumption; (f) progressive periodontitis.

\section{Information collection}

The dental records of participants were reviewed by examiners and the baseline information about patients and implants were obtained. Patient information included age, sex, $\mathrm{HbA1c}$, medication, bone augmentation and oral hygiene care. The implant information included the size, location, arch, position of fixture and major surface treatment of implants. The surgical complications after implant placement were recorded, including wound bleeding, swelling and membrane exposure. And the infection around implant was obtained.

\section{Radiographic analysis}

A standardized digital dental periapical radiographic evaluation performed immediately after implant placement was recorded as the baseline. The radiographic evaluation was carried out before prosthetic installation and at 1-year follow-up after function loading (Figure 1).

All radiographs were viewed on a computer screen using Digimizer v5.4.5 (MedCalc Inc., Mariakerke, Belgium) software. The examiner measured the crestal bone levels as the vertical distance from the tip of the implant body to the coronal edge of the first bone-to-implant contact (Figure 2). The image error of magnification was calibrated by the reference value of the actual implant length.

\section{Clinical parameters at the first year after functional loading}

The clinical parameters recordings at the first year after functional loading were collected and analyzed, including BOP and PD. These clinical parameters were measured at six sites around implants and reported as the mean value.

\section{Statistical analysis}

Statistical analysis was performed using specialized software (SPSS v25.0, IBM, Chicago, Illinois). The baseline information was analyzed by the chi-square test and analysis of variance. Dependent variables were evaluated for a normal distribution by the Shapiro-Wilk test. The data are expressed as the means and SDs. Peri-implant MBL and clinical parameters was compared using the Kruskal-Wallis test. The Bonferroni post hoc test was applied for multiple comparisons. Multiple regression analysis was 
performed to control the baseline variables as covariates. The direction and strength of association between outcome variable and covariates were assessed by regression coefficients, and the precision of which was measured by $95 \%$ confidence intervals. Statistical significance threshold was set at $P<0.05$.

\section{Results}

\section{The general condition of population}

A total of 150 patients with 308 implants with GBR were eligible for this study. The system of implant included Straumann, Basel, Switzerland and Nobel Biocare, Gothenburg, Sweden. The condition of implant was analyzed by position of fixture and the major surface treatment to avoid the conflicts of interest. Sixty-one implants in groups with GBR experiences surgical complication and no implant suffered infection. And 54 patients with 101 implants were followed up for a year after the prosthesis installation, and other patients lost to follow-up. No further complications were reported during the followup period.

\section{The Information Analysis Of Patients And Implants With Gbr}

The basic information about patients and implants with GBR is shown in Table 1. There were 54 patients and 101 implants in insulin group, 54 patients and 121 implants in metformin group, 42 patients and 86 implants in GLP-1 group. These groups were comparable with respect to age, $\mathrm{HbA1c}$, sex, implant location and the major surface treatment of implants $(P>0.05)$. However, the position of fixture was significantly different among the groups $(P<0.01)$. A total of 71 patients with 129 implants completed one-year follow-up after functional loading (30 patients and 53 implants in insulin group, 24 patients and 44 implants in metformin group, 17 patients and 32 implants in GLP-1 group). The basic confounding factors of patients and implants among groups were comparable $(P>0.05)$. 
Table 1

Information on patients and implants with GBR

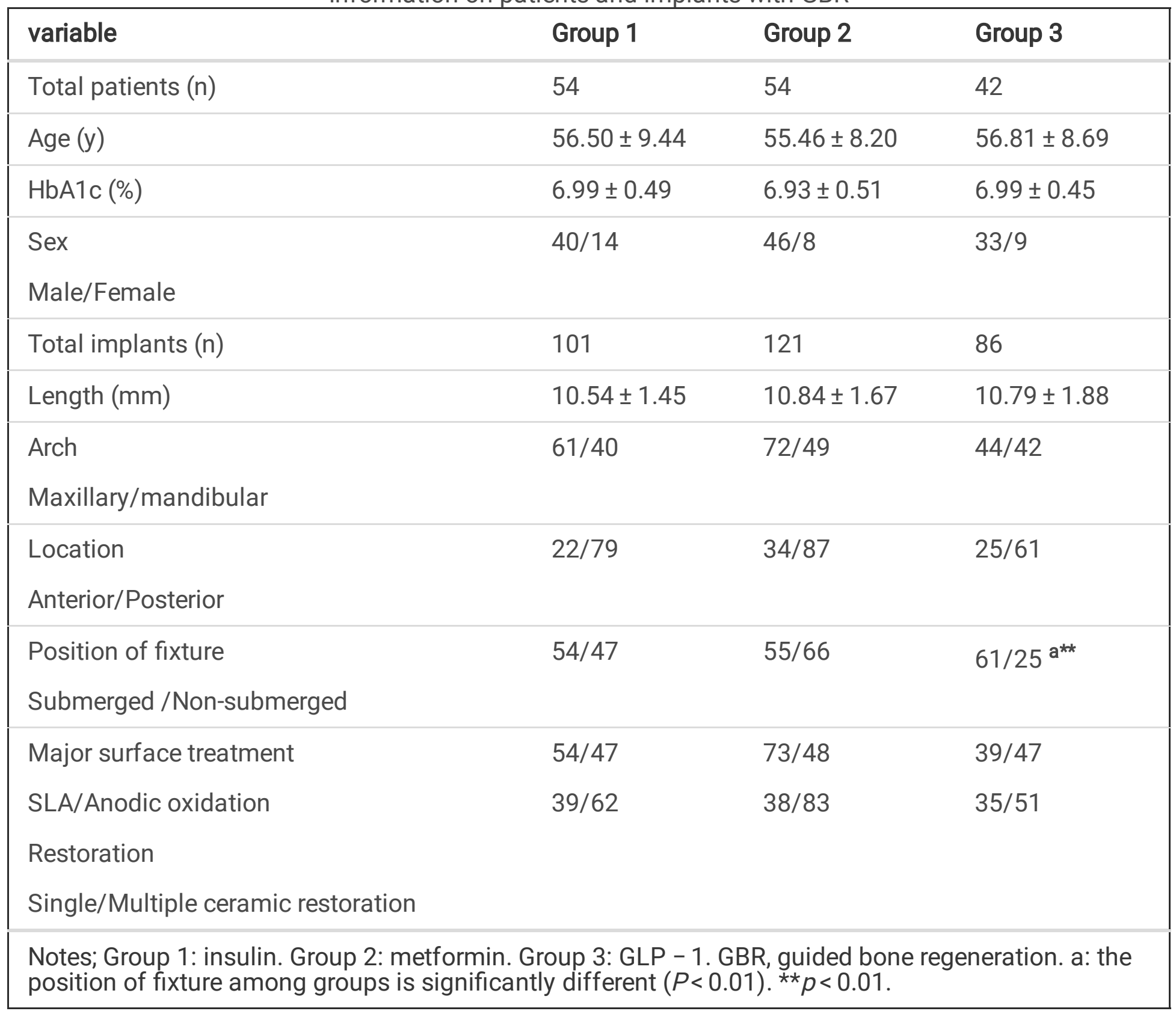

\section{The comparison of radiographic and clinical parameters of implants with GBR}

At 12 months of follow-up, the mean MBL around implants with GBR is shown in Table 2. The MBL was not the same among these medication groups. The mesial and distal MBL parameters in group 1 (mesial: $0.43 \pm 0.14 \mathrm{~mm}$, distal: $0.42 \pm 0.13 \mathrm{~mm})(P<0.05)$ and group 2 (mesial: $0.45 \pm 0.14 \mathrm{~mm}$, distal: $0.47 \pm$ $0.13 \mathrm{~mm})(P<0.01)$ were higher than those in group 3 (mesial: $0.38 \pm 0.12 \mathrm{~mm}$, distal: $0.36 \pm 0.12 \mathrm{~mm})$. The distal MBL was significantly higher in group $2(0.47 \pm 0.13 \mathrm{~mm})$ than in group $1(0.42 \pm 0.13 \mathrm{~mm})(P$ $<0.05)$. Similarly, the distal MBL in the percentage of implant length was significantly higher in group 1 $(P<0.01)$ and group $2(P<0.01)$ than in group 3. While at 24 months of follow-up, the mesial and distal MBL parameters in group 1 (mesial: $0.63 \pm 0.15 \mathrm{~mm}$, distal: $0.61 \pm 0.17 \mathrm{~mm})(P<0.05)$ and group 3 
(mesial: $0.58 \pm 0.13 \mathrm{~mm}$, distal: $0.61 \pm 0.19 \mathrm{~mm})(\mathrm{P}<0.05)$ were smaller than those in group 2 (mesial: $0.72 \pm 0.14 \mathrm{~mm}$, distal: $0.68 \pm 0.13 \mathrm{~mm})$.

Table 2

The comparison of MBL among groups with GBR

\begin{tabular}{|c|c|c|c|c|c|c|}
\hline \multirow[t]{2}{*}{ MBL } & \multicolumn{2}{|l|}{ Group 1} & \multicolumn{2}{|l|}{ Group 2} & \multicolumn{2}{|l|}{ Group 3} \\
\hline & 12 months & 24 months & 12 months & 24 months & 12 months & 24 months \\
\hline $\begin{array}{l}\text { Mesial MBL in } \\
\text { millimeters }\end{array}$ & $\begin{array}{l}0.43 \pm 0.14 \\
\mathrm{a}^{*}\end{array}$ & $\begin{array}{l}0.63 \pm \\
0.15^{c^{\star}}\end{array}$ & $\begin{array}{l}0.45 \pm \\
0.144^{b^{\star \star}}\end{array}$ & $\begin{array}{l}0.72 \pm \\
0.14\end{array}$ & $\begin{array}{l}0.38 \pm \\
0.12\end{array}$ & $\begin{array}{l}0.58 \pm \\
0.13^{\mathrm{d}^{\star \star}}\end{array}$ \\
\hline $\begin{array}{l}\text { Distal MBL in } \\
\text { millimeters }\end{array}$ & $\begin{array}{l}0.42 \pm 0.13 \\
\mathrm{a}^{\star *} \mathrm{C}^{\star}\end{array}$ & $\begin{array}{l}0.61 \pm \\
0.17^{\mathrm{c}^{\star}}\end{array}$ & $\begin{array}{l}0.47 \pm \\
0.13^{b^{* *}}\end{array}$ & $\begin{array}{l}0.68 \pm \\
0.13\end{array}$ & $\begin{array}{l}0.36 \pm \\
0.12\end{array}$ & $\begin{array}{l}0.61 \pm \\
0.19^{c^{\star}}\end{array}$ \\
\hline $\begin{array}{l}\text { Mesial MBL in } \\
\text { percentage }\end{array}$ & $4.08 \pm 1.37$ & $\begin{array}{l}6.05 \pm \\
1.47\end{array}$ & $\begin{array}{l}4.30 \pm \\
1.58^{b^{\star \star}}\end{array}$ & $\begin{array}{l}6.83 \pm \\
1.73\end{array}$ & $\begin{array}{l}3.67 \pm \\
1.50\end{array}$ & $\begin{array}{l}5.48 \pm \\
1.48^{\mathrm{d}^{\star \star}}\end{array}$ \\
\hline $\begin{array}{l}\text { Distal MBL in } \\
\text { percentage }\end{array}$ & $\underset{b * \star}{4.04} \pm 1.25$ & $\begin{array}{l}5.87 \pm \\
1.78\end{array}$ & $\begin{array}{l}4.45 \pm \\
1.566^{\mathrm{b}^{\star \star}}\end{array}$ & $\begin{array}{l}6.48 \pm \\
1.75\end{array}$ & $\begin{array}{l}3.42 \pm \\
1.38\end{array}$ & $\begin{array}{l}5.69 \pm \\
1.97\end{array}$ \\
\hline $\begin{array}{l}\text { Notes; Group } 1 \\
\text { bone regenera } \\
\text { compared with }\end{array}$ & $\begin{array}{l}\text { Group } 2 \\
\text { ompared } \\
2(P<0.0\end{array}$ & $\begin{array}{l}\text { formin. } \mathrm{C} \\
\text { group } 3 \\
\text { compare }\end{array}$ & $\begin{array}{l}\text { 3: GLP } \\
\text { 05), b: c }\end{array}$ & $\begin{array}{l}\text { BL: margi } \\
\text { ared with } \\
0.01) .{ }^{\star} p\end{array}$ & $\begin{array}{l}\text { I bone loss } \\
\text { up } 3(P<0 \\
05, * \star p<0\end{array}$ & $\begin{array}{l}\text { BR, guided } \\
\text { ); } \mathrm{C} \text { : }\end{array}$ \\
\hline
\end{tabular}

After the first year after functional loading, there was no statistical difference in the BOP $(+)(P>0.05)$ and the PD in group $1(1.72 \pm 0.17 \mathrm{~mm})$ was comparable with group $2(1.78 \pm 0.15 \mathrm{~mm})$ and group 3 $(1.69 \pm 0.26 \mathrm{~mm})$ at 24 months of follow-up $(P>0.05)$ (Table 3$)$.

Table 3

The comparison of clinical parameters among groups with GBR

\begin{tabular}{|lllll|}
\hline variables & Group 1 & Group 2 & Group 3 & $P$ \\
\hline $\mathrm{BOP}(+)$ & $44(83.02 \%)$ & $36(81.82 \%)$ & $27(84.38 \%)$ & 0.958 \\
\hline $\mathrm{PD}(\mathrm{mm})$ & $1.72 \pm 0.17$ & $1.78 \pm 0.15$ & $1.69 \pm 0.26$ & 0.071 \\
\hline
\end{tabular}

Notes: Group 1: insulin. Group 2: metformin. Group 3: GLP - 1. BOP: bleeding on probing. PD: probing depth.

\section{Regression analysis to control the position of fixture at the 12 months of follow-up}

The position of fixture among groups should be controlled as confounders by multiple regression analysis (Table 4). The mesial and distal MBL at 12 months of follow-up still showed statistically significant differences among the groups even after controlling for the position of fixture $(P<0.05)$. The table IV showed that the mesial MBL at 12 months of follow-up in group $1(P<0.05)$ and group $2(P<$ $0.01)$ were higher than group 3 and the distal MBL in group $1(P<0.01)$ and group $3(P<0.01)$ were smaller than group 2 after controlling the position of fixture consistent among groups. 
Table 4

MBL in patients with GBR at 12 months of follow-up after controlling the position of fixture

\begin{tabular}{|c|c|c|c|}
\hline Variable & $\beta$ & $(95 \% \mathrm{Cl})$ & $P$ \\
\hline \multicolumn{4}{|l|}{ Mesial MBL in millimeters } \\
\hline Group 1(compared with group 3) & 0.042 & $(0.003,0.080)$ & $0.034^{*}$ \\
\hline Group 2(compared with group 3) & 0.066 & $(0.028,0.104)$ & $0.001^{* *}$ \\
\hline \multicolumn{4}{|l|}{ Distal MBL in millimeters } \\
\hline Group 1 (compared with group 3) & 0.060 & $(0.023,0.097)$ & $0.002^{* *}$ \\
\hline Group 2(compared with group 3) & 0.103 & $(0.067,0.139)$ & $<0.001^{\star \star \star}$ \\
\hline \multicolumn{4}{|l|}{ Distal MBL in millimeters } \\
\hline Group 1 (compared with group 2) & -0.043 & $(-0.077,-0.010)$ & $0.011^{*}$ \\
\hline Group 3(compared with group 2) & -0.103 & $(-0.139,-0.067)$ & $<0.001^{\star \star \star}$ \\
\hline \multicolumn{4}{|l|}{ Mesial MBL in percentage } \\
\hline Group 1 (compared with group 3) & 0.274 & $(-0.147,0.695)$ & 0.201 \\
\hline Group 2(compared with group 3) & 0.436 & $(0.027,0.846)$ & $0.037^{*}$ \\
\hline \multicolumn{4}{|l|}{ Distal MBL in percentage } \\
\hline Group 1(compared with group 3) & 0.485 & $(0.087,0.883)$ & $0.017^{*}$ \\
\hline Group 2(compared with group 3) & 0.841 & $(0.454,1.229)$ & $<0.001^{* \star *}$ \\
\hline \multicolumn{4}{|c|}{$\begin{array}{l}\text { Notes: Group 1: insulin. Group 2: metformin. Group 3: GLP - 1. MBL, marginal bone loss. GBR, guide } \\
\text { bone regeneration. ( } 95 \% \text { CI): } 95 \% \text { confidence interval. P value in bold indicates that the statistical } \\
\text { results are consistent with the results of the Kruskal-Wallis test after accounting for the influence of } \\
\text { covariates on the outcome variable, and the difference among groups is statistically significant. }{ }^{*} p \\
0.05,{ }^{* \star} p<0.01 \text {, }{ }^{* \star} p<0.001\end{array}$} \\
\hline
\end{tabular}

\section{Discussion}

The influence of diabetes on the properties of a patient's bone would impact the outcomes of dental implants. In cases where diabetes cannot be completely cured, clinicians generally use hypoglycemic drugs to reduce the adverse effects of diabetes. Numerous studies have reported the direct effect of hypoglycemic drugs on systemic bone metabolism. However, little attention has been paid to the effect of hypoglycemic drugs on peri-implant bone. In the present study, the peri-implant radiographic parameters showed different characteristics among patients using different hypoglycemic agents. The results could serve as a reference for the direct effect of drugs on systemic bone. 
This study showed that MBL among groups was not exactly the same. The MBL parameters in insulin group and metformin group were higher than those in GLP-1 group at 12 months of follow-up. The MBL in metformin group was higher than insulin group and GLP-1 drugs group at 24 months of follow-up. Similarly, the MBL in the percentage of original implant length in insulin group and metformin group was higher than that in GLP-1 drugs group. During these periods, the bone and bone substitution material had experienced the bone healing and remodeling process and this process could be influenced by the bone condition around implants. The difference of MBL among groups showed the possibility of these drugs interfering with bone tissue around the implant in T2DM patients. After controlling for the baseline information variable, the difference in MBL among groups still existed. This means that these confounding variables, including position of fixture and major surface treatment, did not influence the comparative results of MBL among groups. The change of bone levels around implants could show the bone healing and remodeling process and the higher MBL might suffer higher risk of failure. From these results, the medication type might be related to the bone healing and remodeling around implant at the early stage and the potential among medication might be different. However, the present result showed there was no statistically significant difference in clinical parameters, including BOP (+) and PD, among groups. Although the diabetes could be related to the periodontal inflammation, the peri-implant inflammation parameters seem to have nothing to do with the medication type of the T2DM patient.

The results showed that GLP-1 drugs might have a more positive effect on the peri-implant bone than other agents. This is consistent with previous studies. Liang ${ }^{17}$ indicated that compared with other hypoglycemic drugs, GLP-1 drugs could significantly reduce the risk of fracture. A meta-analysis also supports that exenatide, a GLP-1 receptor agonist (RA), might have advantages in preventing fracture risk over other hypoglycemic drugs ${ }^{18}$. Preclinical studies have reported the superior osteogenic properties of GLP-1 drugs. GLP-1 receptors are widely distributed, and knockout of the GLP-1 receptor gene leads to severe bone changes related to the abnormality of osteoclasts ${ }^{19}$, which supports the direct effect of GLP1 on bone. GLP-1RA might also enhance early osseointegration. GLP-1RA improved factors beneficial to osseointegration around implants in a T2DM animal model ${ }^{20}$. The injection of GLP-1RA could improve serum osteogenesis factors after dental implant surgery ${ }^{21}$. However, some researchers believe that the effect of GLP-1RA on bone is neutral ${ }^{22}$, which may be due to the different eligibility conditions of the study participants. $\mathrm{Li}^{23}$ indicated that there was no difference in the effects of exenatide and insulin on bone turnover markers and bone mineral density, but the observation time was short. More studies are needed to clarify the specific effects of GLP-1RA on bone tissue.

Metformin and insulin are both classic hypoglycemic medications and there are many studies about their positive influence on bone tissue. The results showed that the distal MBL in the metformin group was higher than that in the insulin group $(P<0.05)$. The most previous comparison of the bone metabolism effect between these two drugs focused on the systematic body. Raj ${ }^{24}$ pointed out that compared with metformin, insulin could significantly protect bone through osteocalcin and other pathways. However, some fracture risk studies presented different views. Losada ${ }^{25}$ and Hidayat ${ }^{26}$ determined that metformin could reduce the fracture risk and protect bone metabolism compared with the effects of insulin. The 
underlying mechanism of this contradiction is unclear. The explanation may be that diabetes is generally more severe in insulin users than oral medicine users. Patients using insulin could suffer more fall fractures caused by illness complications and hypoglycemic reactions, while metformin users had a lower incidence of fractures due to fewer complications ${ }^{27}$. Therefore, the fracture risk cannot accurately reflect the influence of drugs on bone metabolism. This study selected patients with good blood glucose control and no severe complications. The interference of different conditions was removed as far as possible to increase the reliability of the results.

There are also many studies on the beneficial bone target mechanism of metformin and insulin. A large number of studies have proven the positive effect of insulin on bone tissue. Insulin has been shown to promote bone formation by upregulating the serum osteogenesis factor ${ }^{28}$. Some studies have shown that a local injection of insulin can promote early fracture healing in diabetic animals ${ }^{29}$. Moreover, local or systematic use of insulin could improve implant osseointegration ${ }^{30-31}$. Another study has shown that insulin could promote angiogenesis ${ }^{32}$, which is also a conducive factor to early osseointegration. Metformin is a first-line drug for the treatment of diabetes. Its positive mechanism on bone has been confirmed by a large number of preclinical studies. It is reported that metformin could promote osteogenesis and inhibit bone resorption ${ }^{33}$. However, metformin would reduce the level of bone turnover factors and hinder the bone remodeling ${ }^{34}$. This effect might compromise the self-repair of minor bone tissue damage. In this study, the MBL in the insulin group was smaller than that in the metformin group, which might be related to their effect on bone metabolism.

At present, the major controversy about the bone target effect of some hypoglycemic drugs entails their indirect effect on bone by controlling blood glucose ${ }^{35}$. Firstly, it is noteworthy that all T2DM patients included in this study had good controlled blood glucose, with $\mathrm{HbA} 1 \mathrm{c}$ less than $8 \%$. And the results showed that MBL was different among groups, even though the patients in each group reported good controlled blood glucose. This meant that these drugs might have different influences on peri-implant bone. The process of bone remodeling around implants requires an environment conducive to bone formation. Therefore, T2DM patients undergoing implant surgery should choose hypoglycemic drugs more conductive to bone formation, such as insulin and GLP-1RA. However, this adjustment in the medication plan should not violate the clinical principle of diabetes treatment. Hence, the results of this study are more useful for patients who have flexibility regarding agent use. One limitation of this study is that blood glucose could not be monitored in real time. Therefore, further laboratory-based investigations should be executed to guarantee blood glucose control more strictly and eliminate bias in this type of research in the future.

Another contribution of the present study is that it provides feedback on clinical systemic bone research. Previous research on the systematic bone target effect of hypoglycemic drugs focused more on the changes in fracture risk, bone mineral density and bone-related biochemical indexes ${ }^{36}$. However, these indexes could not accurately reflect the influence of drugs on bone. The bone mineral density in patients with T2DM might be higher than that in normal individuals, and the fracture risk in T2DM patients could 
be high ${ }^{37}$. The fracture risk reason might be related to damaged bone quality, higher incidence of falls and severe complications of diabetes. While the MBL around the implant is an important criterion for the efficacy of implant treatment and could be affected by bone metabolism ${ }^{38}$. Therefore, MBL could serve as an indicator of the bone target effect and could be used to supplement relevant research results about systematic bone.

Considering the cost and radiation risk, conventional standard periapical radiography is still the most preferable clinical method to assess MBL around implants. This study focused on the MBL around implants with GBR in different hypoglycemic agent groups. However, it is beyond the scope of this study to examine the horizontal change of the alveolar ridge at the implant site as this study was based on twodimensional images. Follow-up studies on the horizontal change in the ridge would be executed by cone beam computed tomography.

\section{Conclusion}

Though some studies have shown the different effects of medication on bone metabolism in T2DM patients, there is still a lack of clinical investigation for peri-implant parameters in T2DM patients with different hypoglycemic agents. This study could offer new reference regarding medication for T2DM patients undergoing implant surgery. The findings of this study suggest different MBL around implants in T2DM patients using different hypoglycemic agents. GLP-1RA seems be beneficial to bone tissue around the implants compared with insulin and metformin. However, the present study could not provide a high level of evidence for the influence of these drugs on bone condition around implants. Because the skeletal effects of hypoglycemic drugs are very complex, and much clinical evidence is still needed through future research.

\section{Abbreviations}

T2DM: type 2 diabetic mellitus

GLP-1: glucagon-like peptide-1

MBL: marginal bone loss

GBR: guided bone regeneration

BOP: bleeding on probing

PD: probing depth

\section{Declarations}

Acknowledgements: Firstly, the authors would like to take this opportunity to show sincere gratitude to Kejian Wu, who has provided so much useful advices on statistical process. Secondly, the authors thank 
the staff in the Department of Oral Implants, School of Stomatology, The Fourth Military Medical University for their help in this project.

Funding: This work was supported by National Natural Science Foundation of China(grant number 81771107)

Competing interests: The authors claim to have no financial interest, either directly or indirectly, in the products or information listed in the article.

Authors' contributions: Shaojie Shi and Yingliang Song conceived the ideas and conception; Feng Ding, Lei Wang and Xingxing Wang collected the data; Shaojie Shi and Feng Ding mearsured the data; Shaojie Shi, Xiangdong Liu and Sijia Zhang analyzed the data; and Shaojie Shi and Lei Wang led the writing. All authors read and approved the final manuscript

Ethics approval and consent to participate: The retrospective cohort study was approved by the Ethics Committee of School of Stomatology, the Fourth Military Medical University (Ethics Approval Number: IRB-REV-2020045) and in compliance with the Helsinki Declaration. All patients were given complete information about the treatment and signed informed consent forms before surgery.

Consent for publication: Not applicable.

Availability of data and materials: The data generated and analyzed during this study are available from corresponding author on reasonable request.

Author details: State key Laboratory of military Stomatology \& National Clinical Research Center for Oral Diseases \& Shaanxi Engineering Research Center for Dental Materials and Advanced Manufacture, Department of Oral Implants, School of Stomatology, The Fourth Military Medical University, Shaanxi Xi'an, 710032, China

\section{References}

1. IDF Diabetes Atlas TEC, IDF DIABETES ATLAS Ninth edition 2019, 2019

2. Glurich I and Acharya A. Updates from the Evidence Base Examining Association between Periodontal Disease and Type 2 Diabetes Mellitus: Current Status and Clinical Relevance. Curr Diabetes Rep 2019; 19.

3. Zhang S, Song $S$ and Wang S, et al. Type 2 diabetes affects postextraction socket healing and influences first-stage implant surgery: A study based on clinical and animal evidence. Clin Implant Dent Relat Res 2019; 21: 436-445.

4. Naenni N, Bienz SP and Muñoz F, et al. Volumetric changes following ridge preservation or spontaneous healing and early implant placement with simultaneous guided bone regeneration. $J$. Clin. Periodontol. 2018; 45: 484-494. 
5. Chambrone $L$ and Palma LF. Current status of dental implants survival and peri-implant bone loss in patients with uncontrolled type-2 diabetes mellitus. Current Opinion in Endocrinology \& Diabetes and Obesity 2019; 26: 219-222.

6. Yamazaki S, Masaki $C$ and Nodai T, et al. The effects of hyperglycaemia on peri-implant tissues after osseointegration. J. Prosthodont Res 2020; 64: 217-223.

7. Shah FA, Thomsen $P$ and Palmquist A. Osseointegration and current interpretations of the boneimplant interface. Acta Biomater 2019; 84: 1-15.

8. Hameed MH, Khan FR and Ghafoor R, et al. Marginal bone loss around cement and screw-retained fixed implant prosthesis. J Clin Exp Dent 2018; 10: e949-e954.

9. Aghaloo T, Pi-Anfruns $\mathrm{J}$ and Moshaverinia A, et al. The Effects of Systemic Diseases and Medications on Implant Osseointegration: A Systematic Review. Int J Oral Maxillofac Implants 2019; 34: s35-s49.

10. Al Amri MD and Abduljabbar TS. Comparison of clinical and radiographic status of platformswitched implants placed in patients with and without type 2 diabetes mellitus: a 24-month follow-up longitudinal study. Clin. Oral Implan. Res. 2017; 28: 226-230.

11. Lagunov VL, Sun J and George R. Evaluation of biologic implant success parameters in type 2 diabetic glycemic control patients versus healthy patients: A meta-analysis. Journal of Investigative and Clinical Dentistry 2019; 10.

12. Kalaitzoglou E, Fowlkes JL and Popescu I, et al. Diabetes pharmacotherapy and effects on the musculoskeletal system. Diabetes/Metabolism Research and Reviews 2019; 35: e3100.

13. Lecka-Czernik B. Diabetes, bone and glucose-lowering agents: basic biology. Diabetologia 2017; 60: 1163-1169.

14. Wang $X$, Qi F and Xing H, et al. Uniform-sized insulin-loaded PLGA microspheres for improved earlystage peri-implant bone regeneration. Drug Deliv. 2019; 26: 1178-1190.

15. Schiellerup SP, Skov-Jeppesen K and Windeløv JA, et al. Gut Hormones and Their Effect on Bone Metabolism. Potential Drug Therapies in Future Osteoporosis Treatment. Front Endocrinol 2019; 10.

16. Ormianer Z, Block J and Matalon S, et al. The Effect of Moderately Controlled Type 2 Diabetes on Dental Implant Survival and Peri-implant Bone Loss: A Long-Term Retrospective Study. The International journal of oral \& maxillofacial implants 2018; 33: 389-394.

17. Cheng L, Hu Y and Li YY, et al. Glucagon-like peptide-1 receptor agonists and risk of bone fracture in patients with type 2 diabetes: A meta-analysis of randomized controlled trials. Diabetes/Metabolism Research and Reviews 2019; 35.

18. Zhang YS, Weng WY and Xie BC, et al. Glucagon-like peptide-1 receptor agonists and fracture risk: a network meta-analysis of randomized clinical trials. Osteoporosis Int. 2018; 29: 2639-2644.

19. Mabilleau G, Gobron B and Bouvard B, et al. Incretin-based therapy for the treatment of bone fragility in diabetes mellitus. Peptides 2018; 100: 108-113. 
20. Liu Z, Zhou W and Tangl S, et al. Potential mechanism for osseointegration of dental implants in Zucker diabetic fatty rats. British Journal of Oral and Maxillofacial Surgery 2015; 53: 748-753.

21. Zhou W, Liu Z and Yao J, et al. The Effects of Exenatide Microsphere on Serum BGP and ALP Levels in ZDF Rats after Implantation. Clin Implant Dent Relat Res 2015; 17: 765-770.

22. Schwartz AV. Diabetes, bone and glucose-lowering agents: clinical outcomes. Diabetologia 2017; 60: 1170-1179.

23. Li R, Xu W and Luo S, et al. Effect of exenatide, insulin and pioglitazone on bone metabolism in patients with newly diagnosed type 2 diabetes. Acta Diabetol. 2015; 52: 1083-1091.

24. Raj J, Venkatachalam S and Shekoba M, et al. Conventional antidiabetic agents and bone health: A pilot case-control study. Perspectives in Clinical Research 2019; 10: 177.

25. Losada E, Soldevila B and Ali MS, et al. Real-world antidiabetic drug use and fracture risk in 12,277 patients with type 2 diabetes mellitus: a nested case-control study. Osteoporosis Int. 2018; 29 : 2079-2086.

26. Hidayat $\mathrm{K}, \mathrm{Du} \mathrm{X}$ and $\mathrm{Wu} \mathrm{MJ}$, et al. The use of metformin, insulin, sulphonylureas, and thiazolidinediones and the risk of fracture: Systematic review and meta-analysis of observational studies. Obes Rev 2019; 20: 1494-1503.

27. JACKULIAK J, KUŽMA M and PAYER J. Effect of Antidiabetic Treatment on Bone. Physiol. Res. 2019: S107-S120.

28. Bortolin RH, Freire NF and Arcaro CA, et al. Anabolic Effect of Insulin Therapy on the Bone: Osteoprotegerin and Osteocalcin Up-Regulation in Streptozotocin-Induced Diabetic Rats. Basic Clin Pharmacol Toxicol 2017; 120: 227-234.

29. Paglia DN, Wey A and Breitbart EA, et al. Effects of local insulin delivery on subperiosteal angiogenesis and mineralized tissue formation during fracture healing. J. Orthop. Res. 2013; 31: 783-791.

30. Malekzadeh BÖ, Erlandsson MC and Tengvall P, et al. Effects of implant-delivered insulin on bone formation in osteoporotic rats. J. Biomed. Mater. Res. A 2018; 106: 2472-2480.

31. Jia T, Wang $Y$ and Zhang J, et al. Cinaciguat in combination with insulin induces a favorable effect on implant osseointegration in type 2 diabetic rats. Biomed. Pharmacother. 2019; 118: 109216.

32. Wang $D$, Du $S$ and $X u M$, et al. Effects of insulin therapy on fracture healing and expression of VEGF in diabetic rats. J. Appl Biomed 2013; 11: 33-40.

33. Serrão CR, Bastos MF and Cruz DF, et al. Role of Metformin in Reversing the Negative Impact of Hyperglycemia on Bone Healing Around Implants Inserted in Type 2 Diabetic Rats. The International journal of oral \& maxillofacial implants 2017; 32: 547-554.

34. Stage TB, Christensen MH and Jørgensen NR, et al. Effects of metformin, rosiglitazone and insulin on bone metabolism in patients with type 2 diabetes. Bone 2018; 112: 35-41.

35. Mabilleau G, Pereira M and Chenu C. Novel skeletal effects of glucagon-like peptide-1 (GLP-1) receptor agonists. J. Endocrinol. 2018; 236: R29-R42. 
36. Miyake H, Kanazawa I and Sugimoto T. Association of Bone Mineral Density, Bone Turnover Markers, and Vertebral Fractures with All-Cause Mortality in Type 2 Diabetes Mellitus. Calcified Tissue Int. 2018; 102: 1-13.

37. Wang $\mathrm{H}, \mathrm{Ba} \mathrm{Y}$ and Xing Q, et al. Diabetes mellitus and the risk of fractures at specific sites: a metaanalysis. Bmj Open 2019; 9: e24067.

38. Kang $\mathrm{M}$, Jung $\mathrm{U}$ and Cho $\mathrm{K}$, et al. Retrospective radiographic observational study of 1692 Straumann tissue-level dental implants over 10 years. II. Marginal bone stability. Clin Implant Dent R. 2018; 20: 875-881.

\section{Figures}

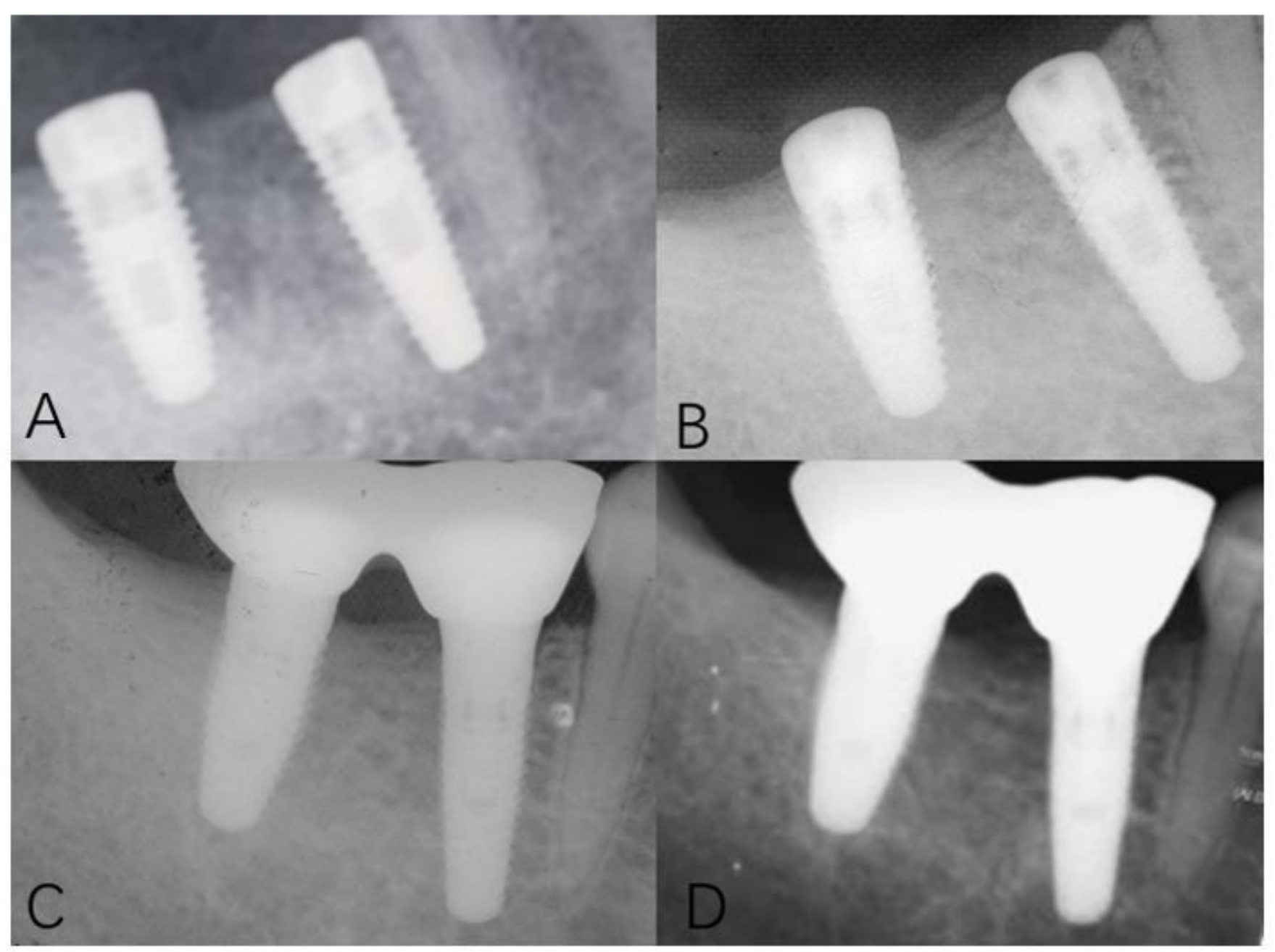

\section{Figure 1}

Periapical radiographs taken. A. Immediately after the first-stage surgery, B. Before the second-stage surgery, C. Immediately after prosthetic installation, D. The first year after functional loading 




Figure 2

The method of measuring the crestal bone levels. 